\title{
On the Evolution of Nearly Circular Vortex Patches
}

\author{
P. Constantin and E. S. Titi \\ Department of Mathematics, University of Chicago, Chicago, IL 60637, USA
}

\begin{abstract}
Recently, the classical problem of the evolution of patches of constant vorticity was reformulated as an evolution equation for the boundary of the patch. We study this equation in the neighborhood of the circular vortex patch and introduce a hierarchy of area-preserving nonlinear approximate equations. The first of these equations is shown to have a rich rigid structure: it possesses an exhaustive increasing sequence of linear invariant manifolds of arbitrarily large finite dimensions. On each of these manifolds the equation can be written as an explicit finite system of ordinary differential equations. Solutions of these ODEs, starting from arbitrarily small neighborhoods of the circular vortex patch, are shown to blow up.
\end{abstract}

\section{Introduction}

Recently, Zabusky et al. [9] and Majda [5] reformulated the classical hydrodynamical problem of the evolution of patches of constant vorticity as an evolution equation for the boundary of the patch. In [5] Majda conjectured that singularities will develop spontaneously in some solutions of the vortex patch equation (VPE). On the other hand, the circular, uniformly rotating vortex patch is a solution of the VPE whose linear marginal stability [4] and even mildly nonlinear stability [7] are well known. Of course, these known stability results are not strong enough to preclude instability and subsequent blow up of quantities such as the length or curvature of vortex patches whose initial shape is nearly circular. In this paper we study the evolution of nearly circular vortex patches. We devise a hierarchy of nonlinear area-preserving approximate equations. These equations intertwine with the equations obtained by retaining finitely many terms in the Taylor expansion near the circle of the nonlinearity. The first of the area-preserving approximate equations, a quadratic nonlocal correction of the linearization about the circular vortex patch, has some interest of its own. For every $n \geqq 0$ the linear manifold of trigonometric polynomials of degree at most $n$ is invariant for this equation. This means that no cascade of "energy" from large scales to small scales occurs: high frequency modes are not excited during the evolution. We obtain blow up results for small data (i.e., nearly circular initial data) for this first approximate equation. 
The paper is organized as follows. In the first section we provide some general considerations on the VPE. One of the motivations for the conjectured blow up of solutions to the VPE was that the equation obtained from the VPE by differentiation is a quadratic integral equation with strongly singular kernel. A similar, but simpler equation was known to blow up ([1]). In Sect. 1 we describe different ways of writing the VPE: one in which the kernel of the integral operator is not singular and another one in which the singular integral operator is well-known (the Hilbert transform).

In Sect. 2 we prove a blow up result for the system obtained from the VPE by differentiating once. The blow up occurs in the absence (and because of the absence) of the constraint that makes the system equivalent to the VPE.

In Sect. 3 we recover the well known Kirchhoff ellipses as exact solutions of the VPE. They are obtained as first order trigonometric polynomials of mean zero. The equations for the coefficients are simple ordinary differential equations which we integrate easily. Section 4 is devoted to the study of the linear approximate equation (linearization) about the circular vortex patch. We find, remarkably, that, although the nonlinearity of the VPE is of high order (requiring, for instance, a Sobolev space of order larger than $3 / 2$ for well-posedness) the first variation of this nonlinearity around the circular vortex patch is a bounded diagonalizable operator in $L^{2}$. We find its explicit expression in the Fourier representation and are able consequently to integrate explicitly the linear approximate equation. The circular vortex patch is found of course to be marginally Lyapunov stable for the linearization. The linearization is an infinite collection of uncoupled $2 \times 2$ complex ordinary differential equations. In Sect. 5 we investigate the area of a patch as a functional of the boundary of the patch. The expression of the area functional in the Fourier representation is

$$
A(z)=\pi \sum_{j=1}^{\infty} j\left(\left|z_{j}\right|^{2}-\left|z_{-j}\right|^{2}\right)
$$

where $z_{j}$ are the Fourier coefficients of the $2 \pi$ periodic curve $\mathrm{z}$.

This shows that the area is a quadratic indefinite functional very similar to the helicity

$$
H(u)=\int \operatorname{curl} u \cdot u d x
$$

for three dimensional incompressible flow. In fact, formula ( 0.1$)$ is exactly the same as the formula expressing the helicity of a flow in terms of its Beltrami decomposition ([2]). We proceed by a proof of the conservation of the area functional for the VPE and for more general singular integro-differential equations. All that is required for the kernel of such an equation in order to obtain the area conservation property is that it be real valued and symmetric. With this observation the task of constructing area preserving approximate equations is easy, not only near the circular vortex patch, but in general. We end Sect. 5 by the study of the first approximate areapreserving equation referred to earlier in this introduction. In Sect. 6 we show that the quadratic (non area-preserving) approximate equation has explicit solutions which do not blow up and approximate well for long times the Kirchhoff solutions. The linear manifolds of trigonometric polynomials of order $n \geqq 4$ are not invariant 
for this equation. However, an infinite dimensional family of exact solutions is found explicitly. Also the manifolds of trigonometric polynomials of order $n \leqq 3$ are found to be invariant and lead to interesting explicit systems of ordinary differential equations.

\section{The Vortex Patch Evolution Equation}

The classical problem of the evolution of patches of constant vorticity is, in modern language, the problem of the evolution of weak solutions of the two-dimensional incompressible Euler equations with initial data prescribed as follows. The initial vorticity is the characteristic function of some set $D_{0}$ in $R^{2}$; the initial velocities are obtained via the familiar Biot-Savart formula.

The global existence and uniqueness of solutions of this problem follow from the fundamental work of Yudovitch ([8]). The vorticity is advected passively and at later time $t$ is the characteristic function of the advected set $D_{t}$. Assuming (for simplicity) $D_{0}$ simply connected and bounded and introducing a parameterization of the boundary $\partial D_{t}$ of $D_{t}$ by a complex valued $2 \pi$ periodic, time dependent function $z(t, \alpha), z(t, \alpha+2 \pi)=z(t, \alpha)$, A. Majda ([5]) derived the following nonlinear equation for the evolution of $z$ :

$$
\frac{\partial z}{\partial t}(t, \alpha)=\frac{1}{2 \pi} \int_{0}^{2 \pi} \log |z(t, \alpha)-z(t, \beta)| \frac{\partial z}{\partial \beta}(t, \beta) d \beta .
$$

Once the curve $z(t, \alpha)$ is known then all the hydrodynamical quantities (vorticity, velocities, pressure) are readily computed. The global estimates of Yudovitch ([8]) for these hydrodynamical quantities are consistent with the possibility of breakdown of solutions of the vortex patch evolution equation (1.1)([5]). The motivation for expecting blow up for the solutions of (1.1) came from two sources: numerical experiments $([9,3])$ and the nature of the equation one obtains by differentiating (1.1). This equation is

with

$$
\frac{\partial \omega}{\partial t}(t, \alpha)=\frac{1}{2 \pi} \int_{0}^{2 \pi} \operatorname{Re}\left(\frac{\omega(t, \alpha)}{z(t, \alpha)-z(t, \beta)}\right) \omega(t, \beta) d \beta,
$$

$$
\omega(t, \alpha)=\frac{\partial z}{\partial \alpha}(t, \alpha) .
$$

The sign $f$ stands for Cauchy's principal value of the integral. Equation (1.2) is a quadratic integral equation with a strongly singular kernel and is similar to the simpler equation

$$
\frac{\partial \omega}{\partial t}(t, \alpha)=\omega(t, \alpha)(H \omega)(t, \alpha)
$$

which does blow up ([1]). Here $H$ is the Hilbert transform. However, unlike in (1.4), the singular kernel in (1.2) depends on the solution via (1.3). This dependence is such that one can rewrite (1.2) in an equivalent form in which the new kernel is nonsingular. In order to show how this is done we recall the well known Plemelj 
formula [6]

$$
\frac{1}{2 \pi i} \underset{\partial D}{f} \frac{f(\zeta) d \zeta}{\zeta-z}=\frac{1}{2} f(z), \quad z \in \partial D
$$

valid for continuous functions in $\partial D$ which can be extended to analytic functions in $D$. Applying Plemelj's formula with $f$ equal to one we can rewrite (1.2) as

$$
\frac{\partial \omega}{\partial t}(t, \alpha)=-\frac{i}{2} \omega(t, \alpha)+\frac{1}{2 \pi i} f_{0}^{2 \pi} \operatorname{Im}\left(\frac{\omega(t, \alpha)}{z(t, \alpha)-z(t, \beta)}\right) \omega(t, \beta) d \beta .
$$

Equation (1.6) has a nonsingular kernel. Indeed, while $\operatorname{Re}((\partial z / \partial \alpha)(t, \alpha) / z(t, \alpha)-z(t, \beta))$ is singular at $\beta=\alpha$, the expression $\operatorname{Im}((\partial z / \partial \alpha)(t, \alpha) / z(t, \alpha)-z(t, \beta))$ has a finite limit as $\beta$ tends to $\alpha$. We will denote throughout this paper by $z_{0}=z_{0}(\alpha)$ the function $e^{i \alpha}$

$$
z_{0}(\alpha)=e^{i \alpha} \text {. }
$$

One can see easily in the form (1.6) of Eq. (1.1) that the unit circular vortex patch rotates with constant angular velocity equal to $-\frac{1}{2}$. That is, the function

$$
z(t, \alpha)=e^{-(i / 2) t} z_{0}(\alpha)
$$

is a solution of (1.1) with initial data equal to $z_{0}$.

In order to have the circular vortex patch as a steady solution we write (1.1) in a frame of reference which rotates together with the unit circular vortex patch. That is, we multiply solutions of (1.1) by $e^{(i / 2) t}$. The new functions, denoted again $z(t, \alpha)$ solve

$$
\frac{\partial z}{\partial t}(t, \alpha)=\frac{i}{2} z(t, \alpha)+\frac{1}{2 \pi} \int_{0}^{2 \pi} \log |z(t, \alpha)-z(t, \beta)| \frac{\partial z}{\partial \beta}(t, \beta) d \beta .
$$

This is true because of the property that, if $z(t, \alpha)$ is a solution of $(1.1)$ then $c z(t, \alpha)$ is also a solution of (1.1) for any $c$ belonging $\mathbb{C}$. This property is of course true for Eq. (1.9), too. It follows that $z_{0}$ is not an isolated steady solution of (1.9); rather, it is imbedded in a complex line $c z_{0}$ of steady solutions. In view of (1.6) we have in this frame of reference the system

$$
\frac{\partial \omega}{\partial t}(t, \alpha)=\frac{1}{2 \pi i} f_{0}^{2 \pi} \operatorname{Im}\left(\frac{\omega(t, \alpha)}{z(t, \alpha)-z(t, \beta)}\right) \omega(t, \beta) d \beta
$$

coupled with

$$
\frac{\partial z}{\partial t}(t, \alpha)=\frac{i}{2} z(t, \alpha)+\frac{1}{2 \pi} \int_{0}^{2 \pi} \log |z(t, \alpha)-z(t, \beta)| \omega(t, \beta) d \beta
$$

and the constraint

$$
\omega(t, \alpha)=\frac{\partial z}{\partial \alpha}(t, \alpha)
$$

It is for solutions of this system, in the absence of (1.12), that we show blow up in the next section. 
Equation (1.1) can be also written as an integral equation in which there are two integral operators: one singular, but linear and with prescribed singular kernel and the other nonlinear, with nonsingular kernel. This can be achieved for any prescribed prototype vortex patch by writing

$$
z(t, \alpha)-z(t, \beta)=\left(\tilde{z}_{0}(\alpha)-\tilde{z}_{0}(\beta)\right)\left(\frac{z(t, \alpha)-z(t, \beta)}{\tilde{z}_{0}(\alpha)-\tilde{z}_{0}(\beta)}\right)
$$

with $\tilde{z}_{0}(\alpha)$ the prototype and using the properties of the logarithm. In particular, for $\tilde{z}_{0}(\alpha)=z_{0}(\alpha)$ we obtain from the form (1.9) of the equation the equivalent form

$$
\frac{\partial z}{\partial t}(t, \alpha)=\frac{1}{2}((i+H) z)(t, \alpha)+\frac{1}{2 \pi} \int_{0}^{2 \pi} \log \left|\frac{z(t, \alpha)-z(t, \beta)}{z_{0}(\alpha)-z_{0}(\beta)}\right| \frac{\partial z}{\partial \beta}(t, \beta) d \beta .
$$

Indeed, this follows from the identity

$$
\frac{1}{2 \pi} \int_{0}^{2 \pi} \log \left|z_{0}(\alpha)-z_{0}(\beta)\right| \frac{\partial f}{\partial \beta}(\beta) d \beta=\frac{1}{2}(H f)(\alpha)
$$

with $H$ the Hilbert transform

$$
(H f)(\alpha)=\frac{1}{2 \pi} f_{0}^{2 \pi} \cot \left(\frac{\alpha-\beta}{2}\right) f(\beta) d \beta .
$$

The form (1.13) of Eq. (1.9) (and thus of Eq. (1.1) in a rotating frame) is best suited for study of nearly circular vortex patches. The relation

$$
(i+H) z_{0}=0
$$

shows again that $z_{0}$ is a steady solution of (1.13) and that (1.13) yields readily an equation for the deviation $\zeta(t, \alpha)=z(t, \alpha)-z_{0}(\alpha)$.

\section{A Blow Up Result}

In this section we consider the system of equations

$$
\left\{\begin{array}{l}
\frac{\partial z}{\partial t}(t, \alpha)=\frac{i}{2} z(t, \alpha)+\frac{1}{2 \pi} \int_{0}^{2 \pi} \log |z(t, \alpha)-z(t, \beta)| \omega(t, \beta) d \beta \\
\frac{\partial \omega}{\partial t}(t, \alpha)=\frac{1}{2 \pi i} \int_{0}^{2 \pi} \operatorname{Im}\left(\frac{\omega(t, \alpha)}{z(t, \alpha)-z(t, \beta)}\right) \omega(t, \beta) d \beta
\end{array}\right.
$$

which, in the presence of the constraint

$$
\omega(t, \alpha)=\frac{\partial z}{\partial \alpha}(t, \alpha)
$$

is equivalent to the equation of evolution of vortex patches (1.13). We will prove a blow up result for the system (2.1) in the absence of the constraint (2.2). Let us seek solutions to (2.1) of the form

$$
\left\{\begin{array}{c}
z(t, \alpha)=\gamma_{1}(t) z_{0}(\alpha) \\
\omega(t, \alpha)=\gamma_{2}(t) \omega_{0}(\alpha)
\end{array}\right.
$$


with $z_{0}(\alpha)=e^{i \alpha}, \omega_{0}(\alpha)=i e^{i \alpha}$. In view of the identity

$$
\frac{i}{2} z_{0}(\alpha)+\frac{1}{2 \pi} \int_{0}^{2 \pi} \log \left|z_{0}(\alpha)-z_{0}(\beta)\right| \frac{\partial z_{0}}{\partial \beta}(\beta) d \beta=0,
$$

we see that the functions $z(t, \alpha), \omega(t, \alpha)$ given by (2.3) solve the first equation of (2.1) provided the coefficients $\gamma_{1}, \gamma_{2}$ satisfy $\dot{\gamma}_{1}=(i / 2)\left(\gamma_{1}-\gamma_{2}\right)$.

Substituting (2.3) in the second equation of (2.1) we obtain

$$
\dot{\gamma}_{2} \omega_{0}(\alpha)=\frac{1}{2 \pi i} \int_{0}^{2 \pi} \operatorname{Im}\left(\frac{\gamma_{2} \omega_{0}(\alpha)}{\gamma_{1}\left(z_{0}(\alpha)-z_{0}(\beta)\right)}\right) \gamma_{2} \omega_{0}(\beta) d \beta .
$$

Now

$$
\frac{\omega_{0}(\alpha)}{z_{0}(\alpha)-z_{0}(\beta)}=\frac{i}{2}+\frac{1}{2} \cot \left(\frac{\alpha-\beta}{2}\right),
$$

and using the identity

$$
\frac{1}{2 \pi} \int_{0}^{2 \pi} \cot \left(\frac{\alpha-\beta}{2}\right) \omega_{0}(\beta) d \beta=-i \omega_{0}(\alpha),
$$

we arrive at the conclusion that $z(t, \alpha), \omega(t, \alpha)$ are solutions of $(2.1)$ if the coefficients $\gamma_{1}, \gamma_{2}$ satisfy the system

$$
\left\{\begin{array}{l}
\dot{\gamma}_{1}=\frac{i}{2}\left(\gamma_{1}-\gamma_{2}\right) \\
\dot{\gamma}_{2}=-\frac{1}{2} \operatorname{Im}\left(\frac{\gamma_{2}}{\gamma_{1}}\right) \gamma_{2} .
\end{array}\right.
$$

Dividing in (2.6) by $\gamma_{1}$ we obtain for

$$
\gamma=\frac{\gamma_{2}}{\gamma_{1}}
$$

the equation

$$
\dot{\gamma}=-\frac{1}{2}(\operatorname{Im} \gamma) \gamma-\frac{i}{2} \gamma(1-\gamma)
$$

We note that if $\gamma_{1}(0)=\gamma_{2}(0)$, i.e., if $\gamma(0)=1$, then for all $t, \gamma_{1}(t)=\gamma_{2}(t)=\gamma_{1}(0)$. This corresponds to solutions of the system (2.1) which satisfy the constraint (2.2) i.e., to solutions of the form $z=c z_{0}, c \in \mathbb{C}$ of the vortex patch evolution equation. However, it is easy to see that if $\operatorname{Re}(\gamma(0))=\frac{1}{3}$, then $\operatorname{Re} \gamma(t)=\frac{1}{3}$ and $\operatorname{Im} \gamma(t)$ becomes $-\infty$ in finite time.

We proved

Theorem 2.1. Solutions of the system (2.1) with initial data of the form

$$
z(0)=\gamma_{1}(0) z_{0}, \quad \omega(0)=\gamma_{2}(0) w_{0},
$$

with $\gamma_{1}(0), \gamma_{2}(0)$ complex numbers are given by

$$
z(t)=\gamma_{1}(t) z_{0}, \quad \omega(t)=\gamma_{2}(t) w_{0}
$$


with $\gamma_{1}(t), \gamma_{2}(t)$ solving $(2.6)$. If

$$
\operatorname{Re}\left(\frac{\gamma_{2}(0)}{\gamma_{1}(0)}\right)=\frac{1}{3}
$$

then these solutions blow up in finite time.

\section{Explicit Solutions of the Vortex Patch Evolution Equation}

The Kirchhoff ellipses are well known exact solutions of the Euler equations ([4]). In this section we recover these solutions in the context of the vortex patch evolution equation. We obtain a parametric representation of the Kirchhoff ellipses as a superposition of $e^{i \alpha}$ and $e^{-i \alpha}$ modes. The coefficients of $e^{i \alpha}$ and $e^{-i \alpha}$ obey simple ordinary differential equations which we integrate easily.

The equation of evolution of vortex patches written in a frame of reference co-rotating with the unit circle is

$$
\frac{\partial z}{\partial t}(t, \alpha)=\frac{1}{2}((i+H) z)(t, \alpha)+\frac{1}{2 \pi} \int_{0}^{2 \pi} \log \left|\frac{z(t, \alpha)-z(t, \beta)}{z_{0}(\alpha)-z_{0}(\beta)}\right| \frac{\partial z}{\partial \beta}(t, \beta) d \beta,
$$

with $z_{0}(\alpha)=e^{i \alpha}$ the unit circle and $H$ the Hilbert transform

$$
(H f)(\alpha)=\frac{1}{2 \pi} f_{0}^{2 \pi} \cot \left(\frac{\alpha-\beta}{2}\right) f(\beta) d \beta .
$$

Because of the identity

$$
(i+H) z_{0}=0,
$$

it is easily seen that $z_{0}$ is a steady solution of (3.1). The explicit Kirchhoff solutions are obtained by seeking $z(t, \alpha)$ of the form

$$
z(t, \alpha)=\left(1+\zeta_{1}(t)\right) e^{i \alpha}+\zeta_{-1}(t) e^{-i \alpha}
$$

with functions $\zeta_{1}(t), \zeta_{-1}(t)$ to be determined.

We compute the integral term in (3.1). The quotient

$$
\frac{z(t, \alpha)-z(t, \beta)}{z_{0}(\alpha)-z_{0}(\beta)}=1+\delta(t, \alpha, \beta)
$$

is given by

$$
\delta(t, \alpha, \beta)=\zeta_{1}(t)-\zeta_{-1}(t) e^{-\imath \alpha} e^{-i \beta} .
$$

We use the expansion of the logarithm

$$
\log |1+\delta|=\operatorname{Re}\left(\sum_{k=1}^{\infty}(-1)^{k+1} \frac{1}{k} \delta^{k}\right)
$$

Raising $\delta(t, \alpha, \beta)$ to a power $k$ we obtain from (3.6),

$$
(\delta(t, \alpha, \beta))^{k}=\zeta_{1}(t)^{k}-\left(k \zeta_{1}(t)^{k-1} \zeta_{-1}(t) e^{-i \alpha}\right) e^{-i \beta}+\cdots
$$


The only nonzero contribution to the integral

$$
\frac{1}{2 \pi} \int_{0}^{2 \pi} \operatorname{Re}\left((-1)^{k+1} \frac{1}{k}(\delta(t, \alpha, \beta))^{k}\right) \frac{\partial z}{\partial \beta}(t, \beta) d \beta
$$

comes from the second term in (3.8). We obtain

$$
\begin{aligned}
& \frac{1}{2 \pi} \int_{0}^{2 \pi} \log |1+\delta(t, \alpha, \beta)| \frac{\partial z}{\partial \beta}(t, \beta) d \beta \\
& =\sum_{k=1}^{\infty} \frac{i}{2}\left(1+\zeta_{1}(t)\right)(-1)^{k}\left(\zeta_{1}(t)\right)^{k-1} \zeta_{-1}(t) e^{-i \alpha} \\
& \quad+\sum_{k=1}^{\infty} \frac{i}{2}\left|\zeta_{-1}(t)\right|^{2} \overline{\left(\zeta_{1}(t)\right)^{k-1}}(-1)^{k-1} e^{i \alpha}
\end{aligned}
$$

Thus

$$
\frac{1}{2 \pi} \int_{0}^{2 \pi} \log \left|\frac{z(t, \alpha)-z(t, \beta)}{z_{0}(\alpha)-z_{0}(\beta)}\right| \frac{\partial z}{\partial \beta}(t, \beta)=\frac{i}{2} \frac{\left|\zeta_{-1}(t)\right|^{2}}{1+\overline{\zeta_{1}(t)}} e^{i \alpha}-\frac{i}{2} \zeta_{-1}(t) e^{-i \alpha}
$$

Adding the linear term we deduce that $z(t, \alpha)$ given by the ansatz (3.4) is a solution of (3.1) if the coefficients $\zeta_{1}(t), \zeta_{-1}(t)$ satisfy the equations

$$
\left\{\begin{array}{c}
\dot{\zeta}_{1}=\frac{i}{2} \frac{\left|\zeta_{-1}\right|^{2}}{1+\bar{\zeta}_{1}} . \\
\dot{\zeta}_{-1}=\frac{i}{2} \zeta_{-1} .
\end{array}\right.
$$

The system (3.10) is readily integrated. We obtain

$$
z(t, \alpha)=\left(1+\zeta_{1}(0)\right) \exp \left\{\frac{i}{2} \frac{\left|\zeta_{-1}(0)\right|^{2}}{\left|1+\zeta_{1}(0)\right|^{2}} t\right\} e^{i \alpha}+\zeta_{-1}(0) e^{\frac{1}{2} t} e^{-i \alpha}
$$

These are the Kirchhoff solutions in a frame co-rotating with the unit circle. It is easily seen that (3.11) represents ellipses. In the original frame of reference the unit circle rotates with angular velocity equal to $-1 / 2$. We obtain the solutions in that frame of reference by multiplying by $e^{-\frac{l}{2} t}$.

\section{Nearly Circular Vortex Patches: The Linear Approximate Equation}

The linear stability of circular vortex patches is well known in the hydrodynamical context ([4]). We compute here explicitly the linearized operator and the general solutions to the linear approximate equation in the framework of the equation of evolution of vortex patches

We consider a smooth periodic function of period $2 \pi$ and expand it in a Fourier series

$$
\zeta(\alpha)=\sum_{j=-\infty}^{\infty} \zeta_{j} e^{i j \alpha}
$$


If the function $\zeta$ is small (in $W^{1, \infty}\left(S^{1}\right)$ for instance) then the expression

$$
E(z)(\alpha)=\frac{1}{2 \pi} \int_{0}^{2 \pi} \log \left|\frac{z(\alpha)-z(\beta)}{z_{0}(\alpha)-z_{0}(\beta)}\right| \frac{\partial z}{\partial \beta}(\beta) d \beta
$$

for $z(\alpha)=e^{i \alpha}+\zeta(\alpha)$ can be computed integrating term by term in the series

$$
\sum_{k=1}^{\infty}(-1)^{k+1} \frac{1}{k} \frac{1}{2 \pi} \int_{0}^{2 \pi}\left[\operatorname{Re}\left(\left(\frac{\zeta(\alpha)-\zeta(\beta)}{z_{0}(\alpha)-z_{0}(\beta)}\right)^{k}\right)\right] \frac{\partial z}{\partial \beta}(\beta) d \beta .
$$

Retaining only the first order terms in $\zeta$ we obtain the expression

$$
E_{z_{0}}^{\prime}(\zeta)=\frac{i}{2 \pi} \int_{0}^{2 \pi} \operatorname{Re}\left(\frac{\zeta(\alpha)-\zeta(\beta)}{z_{0}(\alpha)-z_{0}(\beta)}\right) e^{i \beta} d \beta
$$

This expression can be obtained, of course, by formally differentiating $E(z)$ at $z_{0}$ in the direction $\zeta$.

Now, with (4.1) we have

$$
\delta(\alpha, \beta)=\frac{\zeta(\alpha)-\zeta(\beta)}{z_{0}(\alpha)-z_{0}(\beta)}=\sum_{j=-\infty}^{\infty} \zeta_{j} \frac{e^{i j \alpha}-e^{i j \beta}}{e^{i \alpha}-e^{i \beta}} .
$$

We introduce the notation

$$
Q_{j}(\alpha, \beta)=\frac{e^{i j \alpha}-e^{i j \beta}}{e^{i \alpha}-e^{i \beta}}
$$

Thus

$$
\delta(\alpha, \beta)=\sum_{j=-\infty}^{\infty} \zeta_{j} Q_{j}(\alpha, \beta)
$$

Now, for $Q_{j}(\alpha, \beta)$ we have the obvious formulae:

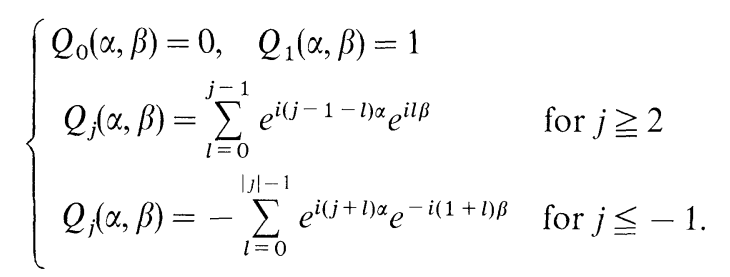

Integrating we obtain

$$
\frac{i}{2 \pi} \int_{0}^{2 \pi} \operatorname{Re}(\delta(\alpha, \beta)) e^{i \beta} d \beta=\sum_{j=-\infty}^{-1}\left(-\frac{i}{2}\right) \zeta_{j} e^{i j \alpha}+\sum_{j=2}^{\infty}\left(\frac{i}{2}\right) \bar{\zeta}_{j} e^{i(2-j) \alpha}
$$

This means that

$$
E_{z_{0}}^{\prime}(\zeta)=\frac{i}{2} \bar{\zeta}_{2}+\frac{i}{2} \sum_{j=-\infty}^{-1}\left(\bar{\zeta}_{2-j}-\zeta_{j}\right) e^{i j \alpha}
$$

On the other hand the Fourier representation of the linear operator $\frac{1}{2}(i+H)$ is

$$
\frac{1}{2}(i+H) \zeta=\frac{i}{2} \zeta_{0}+i \sum_{j=-\infty}^{-1} \zeta_{j} e^{i j \alpha}
$$


Adding we obtain

$$
\frac{1}{2}(i+H) \zeta+E_{z_{0}}^{\prime}(\zeta)=\frac{i}{2} \sum_{j=-\infty}^{0}\left(\zeta_{j}+\bar{\zeta}_{2-j}\right) e^{i j \alpha} .
$$

Let us consider the expression

$$
N(z)(\alpha)=\frac{1}{2 \pi} \int_{0}^{2 \pi} \log |z(\alpha)-z(\beta)| \frac{\partial z}{\partial \beta}(\beta) d \beta+\frac{i}{2} z(\alpha) .
$$

As mentioned in the introduction, because of the fact that

we have

$$
\frac{1}{2 \pi} \int_{0}^{2 \pi} \log \left|z_{0}(\alpha)-z_{0}(\beta)\right| \frac{\partial z}{\partial \beta} d \beta=\frac{1}{2}(H z)(\alpha),
$$

$$
N(z)=\frac{1}{2}(i+H) z+E(z) .
$$

Thus the Gateaux derivative of $N(z)$ at $z=z_{0}$ is

$$
N_{z_{0}}^{\prime}(\zeta)=\frac{1}{2}(i+H) \zeta+E_{z_{0}}^{\prime}(\zeta)
$$

and is given by (4.8).

Note the very remarkable fact that although $N(z), E(z)$ are not continuous or even defined in a $L^{2}$ neighbourhood of $z_{0}$ their Gateaux derivatives are bounded linear operators in $L^{2}$.

The equation of evolution of vortex patches is

$$
\frac{d z}{d t}=N(z)
$$

with $N(z)$ given in (4.9). It is a meaningful equation in a neighborhood of $z_{0}$ in the Sobolev space $H^{2}\left(S^{1}\right)=W^{2,0}\left(S^{1}\right)$. The linear approximate equation (linearization) around $z_{0}$ is $\frac{d \zeta}{d t}=N_{z_{0}}^{\prime}(\zeta)$ i.e.,

$$
\frac{d \zeta}{d t}(t, \alpha)=\frac{1}{2}((i+H) \zeta)(t, \alpha)+\frac{i}{2 \pi} \int_{0}^{2 \pi} \operatorname{Re}\left(\frac{\zeta(t, \alpha)-\zeta(t, \beta)}{z_{0}(\alpha)-z_{0}(\beta)}\right) e^{i \beta} d \beta .
$$

It makes sense if $\zeta$ is a $L^{2}\left(S^{1}\right)$ valued function.

Theorem 4.1. (i) The Gateaux derivative $N_{z_{0}}^{\prime}$ at $z_{0}=e^{i \alpha}$ of the functional

$$
N(z)(\alpha)=\frac{i}{2} z(\alpha)+\frac{1}{2 \pi} \int_{0}^{2 \pi} \log |z(\alpha)-z(\beta)| \frac{\partial z}{\partial \beta}(\beta) d \beta
$$

is a bounded, diagonalizable linear operator in $L^{2}\left(S^{1}\right)$. Its spectrum consists of the two numbers 0 and $i / 2$. (ii) The linear approximate equation

$$
\frac{d \zeta}{d t}=N_{z_{0}}^{\prime}(\zeta)
$$

is equivalent to the uncoupled infinite collection of ordinary differential equations 


$$
\left\{\begin{array}{l}
\dot{\zeta}_{1}=0 \text { and } \\
\dot{\zeta}_{2-j}=0 \\
\dot{\zeta}_{j}=\frac{i}{2}\left(\zeta_{j}+\bar{\zeta}_{2-j}\right) \text { for } j=0,-1,-2, \ldots
\end{array}\right.
$$

The explicit solutions of the linear approximate equation

$$
\zeta(t, \alpha)=\sum_{j=-\infty}^{\infty} \zeta_{j}(t) e^{i j \alpha}
$$

with $\zeta_{j}(t)$ given by

$$
\left\{\begin{array}{l}
\zeta_{j}(t)=\zeta_{j}(0) \quad \text { for } j \geqq 1 \\
\zeta_{j}(t)=-\bar{\zeta}_{2-j}(0)+\left(\zeta_{j}(0)+\bar{\zeta}_{2-j}(0)\right) e^{\frac{2}{2} t}, \quad \text { for } j \leqq 0
\end{array}\right.
$$

show that $\zeta=c z_{0}, c \in \mathbb{C}$, are Lyapunov stable solutions.

Proof. A complete set of eigenfunctions for $N_{z_{0}}^{\prime}$ is, in view of (4.8), (4.12), the collection $e^{i j \alpha}, j \leqq 0$ for the eigenvalue $i / 2$ and $e^{i \alpha}, e^{i k \alpha}-e^{i(2-k) \alpha}, k \geqq 2$ for the eigenvalue 0 . The proof of (ii) follows from (4.8), (4.12) by straightforward computation.

\section{Area Preserving Equations}

The area of a vortex patch is conserved in time; this follows from well known properties of solutions of incompressible Euler equations.

Moreover, in [7] a result of nonlinear stability of nearly circular vortex patches in the sense of the area is proven. In this section we formulate the area in terms of the Fourier expansion of the boundary of a vortex patch. We proceed to describe a hierarchy of equations which preserve area and approximate the vortex patch evolution equation. The first of these equations is shown to possess invariant manifolds of arbitrarily large finite dimension.

If the boundary $\Gamma$ of a domain in $\mathbb{C}$ is described parametrically by

$$
\Gamma=\{z \in \mathbb{C} \mid z=z(\alpha), \alpha \in[0,2 \pi]\},
$$

where the smooth periodic curve $z(\alpha)$ runs through $\Gamma$ counterclockwise as $\alpha$ increases, then the area of the domain is given by

$$
\tilde{A}=\frac{1}{2} \int_{0}^{2 \pi} \operatorname{Im}\left(\frac{\partial z}{\partial \alpha}(\alpha) \bar{z}(\alpha)\right) d \alpha .
$$

We normalize $\tilde{A}$ such that the area of the unit circle is one, $A=(1 / \pi) \tilde{A}$ :

$$
A(z)=\frac{1}{2 \pi} \int_{0}^{2 \pi} \operatorname{Im}\left(\frac{\partial z}{\partial \alpha}(\alpha) \bar{z}(\alpha)\right) d \alpha .
$$

If the Fourier series of the function $z$ is

$$
z(\alpha)=\sum_{j=-\infty}^{\infty} z_{j} e^{i j \alpha}
$$


it follows that

$$
A(z)=\sum_{j=-\infty}^{\infty} j\left|z_{j}\right|^{2}
$$

Note that A is not positive definite. Negative area can occur (for instance through change of orientation such as in the case of $\left.e^{-i \alpha}\right)$. The area functional is well defined for functions $z \in H^{1 / 2}\left(S^{1}\right)$. One can rewrite (5.4) as

$$
A(z)=\sum_{j=1}^{\infty} j\left(\left|z_{j}\right|^{2}-\left|z_{-j}\right|^{2}\right) .
$$

One is reminded, when one looks at (5.5), of the formula for the helicity of an incompressible three dimensional flow expressed in terms of its Beltrami decomposition ([2]). We note that, as long as there are only positive or only negative modes present, the conservation of the area implies boundedness of the $H^{1 / 2}\left(S^{1}\right)$ norm (if $z_{0}$ is bounded)

$$
\|z\|_{1 / 2}=\left(\sum_{j=-\infty}^{\infty}|j|\left|z_{j}\right|^{2}+\left|z_{0}\right|^{2}\right)^{1 / 2} .
$$

But if both negative and positive modes are present then area conservation does not prevent growth of the $H^{1 / 2}$ norm. Of course, higher order norms can grow in either case.

Now we are going to present the proof of conservation of area in the context of the vortex patch evolution equation. From this proof it will be clear how to approximate the vortex patch evolution equation in a way that preserves area.

Let $z(t, \alpha)$ be a solution of the vortex patch equation

$$
\frac{\partial z}{\partial t}(t, \alpha)=\frac{1}{2}((i+H) z)(t, \alpha)+\frac{1}{2 \pi} \int_{0}^{2 \pi} \log \left|\frac{z(t, \alpha)-z(t, \beta)}{z_{0}(\alpha)-z_{0}(\beta)}\right| \frac{\partial z}{\partial \beta}(t, \beta) d \beta .
$$

Then $A(z(t, \cdot))$ evolves according to

$$
\frac{d}{d t} A(z(t, \cdot))=\frac{2}{2 \pi} \int_{0}^{2 \pi} \operatorname{Im}\left(\frac{\partial \bar{z}}{\partial t}(t, \alpha) \frac{\partial z}{\partial \alpha}(t, \alpha)\right) d \alpha .
$$

Using the fact that $H \frac{\partial}{\partial \alpha}$ is selfadjoint we deduce

$$
\begin{aligned}
\frac{d}{d t} A(z(t, \cdot))= & \frac{1}{2 \pi} \int_{0}^{2 \pi} \frac{d}{d \alpha}|z(t, \alpha)|^{2} d \alpha \\
& +\frac{1}{2 \pi^{2}} \int_{0}^{2 \pi} \int_{0}^{2 \pi} \log \left|\frac{z(t, \alpha)-z(t, \beta)}{z_{0}(\alpha)-z_{0}(\beta)}\right| \operatorname{Im}\left(\frac{\partial z}{\partial \alpha}(t, \alpha) \frac{\partial \bar{z}}{\partial \beta}(t, \beta)\right) d \alpha d \beta .
\end{aligned}
$$

Because of the periodicity of $z(t, \alpha)$ we have

$$
\frac{d}{d t} A(z(t, \cdot))=\frac{1}{2 \pi^{2}} \int_{0}^{2 \pi} \int_{0}^{2 \pi} \log \left|\frac{z(t, \alpha)-z(t, \beta)}{z_{0}(\alpha)-z_{0}(\beta)}\right| \operatorname{Im}\left(\frac{\partial z}{\partial \alpha}(t, \alpha) \frac{\partial \bar{z}}{\partial \beta}(t, \beta)\right) d \alpha d \beta .
$$

Now, the expression $\log \left|(z(t, \alpha)-z(t, \beta)) /\left(z_{0}(\alpha)-z_{0}(\beta)\right)\right|$ is symmetric in $\alpha, \beta$ 
while the expression $\operatorname{Im}(\partial z / \partial \alpha(t, \alpha) \partial \bar{z} / \partial \beta(t, \beta))$ is antisymmetric. The integral must vanish. We have actually proven the following general observation:

Proposition 5.1. Let the smooth periodic function $z(t, \alpha)$ solve an equation of the type

$$
\frac{\partial z}{\partial t}(t, \alpha)=\frac{1}{2}((i+H)(z))(t, \alpha)+\frac{1}{2 \pi} \int_{0}^{2 \pi} K(\alpha, \beta, z(t, \alpha), z(t, \beta)) \frac{\partial z}{\partial \beta}(t, \beta) d \beta,
$$

where the kernel $K\left(\alpha, \beta, z_{1}, z_{2}\right)$ is real valued and symmetric: $K\left(\alpha, \beta, z_{1}, z_{2}\right)=$ $K\left(\beta, \alpha, z_{2}, z_{1}\right)$. Then the area of the region enclosed by the curve $z(t, \cdot)$ is conserved:

$$
A(z(t, \cdot))=A(z(0, \cdot)) \text {. }
$$

Let us emphasize that the linear term and the integral term in the right-hand side of (5.9) preserve area separately.

If one wants to build approximate equations for the vortex patch equation one can use either the form (5.7) of the equation or the original form, in which the term

$$
\frac{1}{2}(H z)(\alpha)=\frac{1}{2 \pi} \int_{0}^{2 \pi} \log \left|z_{0}(\alpha)-z_{0}(\beta)\right| \frac{\partial z}{\partial \beta} d \beta
$$

is not pulled out. We prefer the form (5.7) because we concentrate on nearly circular vortex patches. But one can use any fixed function $\tilde{z}_{0}(\alpha)$ instead of $z_{0}(\alpha)=e^{i \alpha}$ as a prototype vortex patch and write

$$
\log |z(t, \alpha)-z(t, \beta)|=\log \left|\tilde{z}_{0}(\alpha)-\tilde{z}_{0}(\beta)\right|+\log \left|\frac{z(t, \alpha)-z(t, \beta)}{\tilde{z}_{0}(\alpha)-\tilde{z}_{0}(\beta)}\right| .
$$

Then in the expansion

$$
\log \left|\frac{z(t, \alpha)-z(t, \beta)}{\tilde{z}_{0}(\alpha)-\tilde{z}_{0}(\beta)}\right|=\operatorname{Re} \sum_{k=1}^{\infty}(-1)^{k+1} \frac{1}{k}\left(\frac{\left(z-\tilde{z}_{0}\right)(\alpha)-\left(z-\tilde{z}_{0}\right)(\beta)}{\tilde{z}_{0}(\alpha)-\tilde{z}_{0}(\beta)}\right)^{k}
$$

one can stop at some level $n$; the approximating kernels

$$
\tilde{K}_{n}\left(\alpha, \beta, z_{1}, z_{2}\right)=\log \left|\tilde{z}_{0}(\alpha)-\tilde{z}_{0}(\beta)\right|+\operatorname{Re} \sum_{k=1}^{n}(-1)^{k+1} \frac{1}{k}\left(\frac{z_{1}-z_{2}}{\tilde{z}_{0}(\alpha)-\tilde{z}_{0}(\beta)}-1\right)^{k}
$$

give rise to area preserving equations.

Thus, for arbitrary initial datum $\tilde{z}_{0}(\alpha)$ one can approximate for short times the vortex patch equation (1.1) by an area preserving equation.

$$
\begin{aligned}
& \frac{\partial z^{(n)}}{\partial t}(t, \alpha)= \frac{1}{2 \pi} \int_{0}^{2 \pi} \log \left|\tilde{z}_{0}(\alpha)-\tilde{z}_{0}(\beta)\right| \frac{\partial z^{(n)}}{\partial \beta}(t, \beta) \\
&+\sum_{k=1}^{n}(-1)^{k+1} \frac{1}{k} \frac{1}{2 \pi} \int_{0}^{2 \pi} \operatorname{Re}\left(\left(\frac{z^{(n)}(t, \alpha)-z^{(n)}(t, \beta)}{\tilde{z}_{0}(\alpha)-\tilde{z}_{0}(\beta)}-1\right)^{k}\right) \frac{\partial z^{(n)}}{\partial \beta}(t, \beta) d \beta \\
& z^{(n)}(0, \alpha)=\tilde{z}_{0}(\alpha)
\end{aligned}
$$


After integrating for short time in (5.11) one can replace $\tilde{z}_{0}(\alpha)$ by $z^{(n)}(t, \alpha)$ and repeat the procedure. One obtains thus a method of approximating the vortex patch equation by nonlinear integro-differential equations with kernels with known singularities and with exact conservation of area.

Let us return now to the nearly circular vortex patches.

Using the notation

$$
\delta(t, \alpha, \beta)=\frac{z(t, \alpha)-z(t, \beta)}{z_{0}(\alpha)-z_{0}(\beta)}-1,
$$

we can write the $n^{\text {th }}$ equation in the hierarchy of area preserving equations as

$$
\frac{\partial z}{\partial t}(t, \alpha)=\frac{1}{2}((i+H) z)(t, \alpha)+\sum_{k=1}^{n}(-1)^{k+1} \frac{1}{k} \frac{1}{2 \pi} \int_{0}^{2 \pi} \operatorname{Re}\left(\delta(t, \alpha, \beta)^{k}\right) \frac{\partial z}{\partial \beta}(t, \beta) d \beta
$$

The first of these equations is

$$
\frac{\partial z}{\partial t}(t, \alpha)=\frac{1}{2}((i+H) z)(t, \alpha)+\frac{1}{2 \pi} \int_{0}^{2 \pi} \operatorname{Re}(\delta(t, \alpha, \beta)) \frac{\partial z}{\partial \beta}(t, \beta) d \beta .
$$

Introducing

Eq. (5.14) becomes

$$
z(t, \alpha)-z_{0}(\alpha)=\zeta(t, \alpha)
$$

$$
\frac{\partial \zeta}{\partial t}(t, \alpha)=\frac{1}{2}((i+H) \zeta)(t, \alpha)+\frac{1}{2 \pi} \int_{0}^{2 \pi} \operatorname{Re}\left(\frac{\zeta(t, \alpha)-\zeta(t, \beta)}{z_{0}(\alpha)-z_{0}(\beta)}\right) \frac{\partial}{\partial \beta}\left(z_{0}+\zeta\right)(t, \beta) d \beta .
$$

Note that (5.15) differs from the linear approximate equation (4.14) by the quadratic term

$$
\frac{1}{2 \pi} \int_{0}^{2 \pi} \operatorname{Re}\left(\frac{\zeta(t, \alpha)-\zeta(t, \beta)}{z_{0}(\alpha)-z_{0}(\beta)}\right) \frac{\partial \zeta}{\partial \beta}(t, \beta) d \beta
$$

In general, the $n^{\text {th }}$ equation (5.13) equals the $n^{\text {th }}$ order Taylor expansion equation plus the $(n+1)^{\text {th }}$ order correction term

$$
(-1)^{n+1} \frac{1}{n} \frac{1}{2 \pi} \int_{0}^{2 \pi} \operatorname{Re}\left(\delta^{n}(t, \alpha, \beta)\right) \frac{\partial \zeta}{\partial \beta}(t, \beta) d \beta .
$$

Thus the equations obtained by expanding the nonlinear term in Taylor series and turncating at some order $n$ need an $n+1$ order correction term in order to become area preserving. In particular, the linear approximate equation (4.14) is not area preserving. This can also be checked on the explicit solutions (4.16).

In the remainder of this section we will investigate the equation (5.15).

First we state the well posedness (local existence) result.

Proposition 5.2. Let $s>\frac{3}{2}$. Assume $\zeta(0) \in H^{s}\left(S^{1}\right)$. Then there exists a unique solution $\zeta(t)$ of Eq. (5.15) with initial datum $\zeta(0)$, for $|t| \leqq T_{0}$ with $T_{0}$ depending on the norm of $\zeta(0)$ in $H^{s}\left(S^{1}\right)$. 
The proof of Proposition 5.2 follows using standard techniques and the following

Lemma 5.3. Let $f$ and $g$ be smooth complex valued periodic functions of period $2 \pi$. Let $b(f, g)$ be defined by

$$
b(f, g)(\alpha)=\frac{1}{2 \pi} \int_{0}^{2 \pi} \operatorname{Re}\left(\frac{f(\alpha)-f(\beta)}{z_{0}(\alpha)-z_{0}(\beta)}\right) \frac{\partial g}{\partial \beta}(\beta) d \beta .
$$

Then, for every $s \geqq 0$

$$
\|b(f, g)\|_{s} \leqq c_{s}[g]\|f\|_{s}
$$

with

$$
[g]=\sum_{j=-\infty}^{\infty}|j|\left|g_{j}\right|, \quad g_{j}=\frac{1}{2 \pi} \int_{0}^{2 \pi} g(\alpha) e^{-i j \alpha} d \alpha, \quad\|f\|_{s}=\left(\left|f_{0}\right|^{2}+\sum_{j=-\infty}^{\infty}|j|^{2 s}\left|f_{j}\right|^{2}\right)^{1 / 2} .
$$

In particular, if $s>\frac{3}{2}$

$$
\|b(f, g)\|_{s} \leqq \tilde{c}_{s}\|g\|_{s}\|f\|_{s} .
$$

Proof. Let us set

$$
c=b(f, g)
$$

and develop

and

$$
f(\alpha)=\sum_{j=-\infty}^{\infty} f_{j} e^{i j \alpha}, \quad g(\alpha)=\sum_{j=-\infty}^{\infty} g_{j} e^{i j \alpha}
$$

Now

$$
c(\alpha)=\sum_{j=-\infty}^{\infty} c_{j} e^{i j \alpha}
$$

$$
\frac{f(\alpha)-f(\beta)}{z_{0}(\alpha)-z_{0}(\beta)}=\sum_{j=-\infty}^{\infty} f_{j} Q_{j}(\alpha, \beta)
$$

with $Q_{j}(\alpha, \beta)$ given in (4.2). With some amount of work we arrive at the expressions for $c_{j}$ :

$$
\left\{\begin{array}{l}
c_{j}=-\frac{i}{2} \sum_{k=-\infty}^{j}(j+1-k) g_{j+1-k} f_{k}+\frac{i}{2} \sum_{k=-j+1}^{\infty}(k+j-1) g_{k+j-1} \bar{f}_{k}, \text { for } j \leqq-1 \\
c_{0}=\frac{i}{2} \sum_{k=1}^{\infty}\left((1-k) g_{1-k} f_{k}+(k-1) g_{k-1} \bar{f}_{k}\right), \text { and } \\
c_{j}=-\frac{i}{2} \sum_{k=-\infty}^{-j}(j+k-1) g_{j+k-1} \bar{f}_{k}+\frac{i}{2} \sum_{k=j+1}^{\infty}(j+1-k) g_{j+1-k} f_{k}, \text { for } j \geqq 1 .
\end{array}\right.
$$

The important property of these expressions is that in each of the sums appearing in (5.19) the range of summation in the dummy variable $k$ is included in 
the tail

$$
|k| \geqq|j| \text {. }
$$

In order to prove the estimate we proceed by duality: we prove that for every sequence of numbers $d_{j}$ such that $\sum_{j=-\infty}^{\infty}\left|d_{j}\right|^{2}=1$, we have the estimate

$$
\left.\left|\sum_{j=-\infty}^{\infty}\right| j\right|^{s}\left|c_{j}\right|\left|d_{j}\right|+\left|c_{0}\right|\left|d_{0}\right| \mid \leqq c_{s}\|f\|_{s}[g] .
$$

In each expression for $c_{j}$ we majorize $\left|c_{j}\right|$ by the corresponding sum of absolute values. To exemplify let us take one of the expressions and use $|j| \leqq|k|$ in it:

$$
\begin{aligned}
& \sum_{j=-\infty}^{-1}|j|^{s}\left|d_{j}\right| \sum_{k=-\infty}^{j}\left|(j+1-k)\left\|g_{j+1-k}\right\| f_{k}\right| \\
& \leqq \sum_{j=-\infty}^{-1}\left(\sum_{k=-\infty}^{j}|k|^{s}\left|f_{k}\|j+1-k\| g_{j+1-k}\right|\right)\left|d_{j}\right| \\
& =\sum_{l=1}^{\infty}|l|\left|g_{l}\right|\left(\sum_{k=-\infty}^{l-1}|k|^{s}\left|f_{k}\right|\left|d_{l+k-1}\right|\right) \\
& \leqq\left(\sum_{l=1}^{\infty}|l|\left|g_{l}\right|\right)\|f\|_{s} .
\end{aligned}
$$

The other terms are dealt with in an entirely similar way. This concludes the proof of the lemma.

The expression (5.19) can be used to derive the equivalent form of the Eq. (5.15) written as an infinite system of ordinary differential equations for the coefficients

$$
\begin{aligned}
& \zeta_{j}=1 / 2 \pi \int_{0}^{2 \pi} \zeta(t, \alpha) e^{-i j \alpha} d \alpha: \\
& \left\{\begin{aligned}
\dot{\zeta}_{j}= & \frac{1}{2}\left(\zeta_{j}+\bar{\zeta}_{2-j}\right)-\frac{i}{2} \sum_{k=-\infty}^{j}(j+1-k) \zeta_{j+1-k} \zeta_{k} \\
& +\frac{i}{2} \sum_{k=-j+1}^{\infty}(k+j-1) \zeta_{k+j-1} \bar{\zeta}_{k}, \text { for } j \leqq-1, \\
\dot{\zeta}_{0}= & \frac{i}{2}\left(\zeta_{0}+\bar{\zeta}_{2}\right)+\frac{i}{2} \sum_{k=1}^{\infty}(1-k) \zeta_{1-k} \zeta_{k}+\frac{i}{2} \sum_{k=1}^{\infty}(k-1) \zeta_{k-1} \bar{\zeta}_{k} \text { and } \\
\dot{\zeta}_{j}= & -\frac{i}{2} \sum_{k=-\infty}^{-j}(j+k-1) \zeta_{j+k-1} \bar{\zeta}_{k}+\frac{i}{2} \sum_{k=j+1}^{\infty}(j+1-k) \zeta_{j+1-k} \zeta_{k}, \text { for } j \geqq 1
\end{aligned}\right.
\end{aligned}
$$

The remark that the range of the dummy variable $k$ is, for each $j$, included in the tail $|k| \geqq|j|$, which was used in the proof of Lemma 5.3 indicates that the nonlinearity in Eq. (5.15) has a very special nature: there is no cascade of "energy" from large scales to small scales. More precisely, if the initial datum $\zeta(0)$ has Fourier 
coefficients of finite support:

$$
\zeta_{j}(0)=0 \text { for }|j| \geqq n+1, \quad n \geqq 0
$$

then the solution $\zeta(t, \alpha)$ has Fourier coefficients with the same property

$$
\zeta_{j}(t)=0 \text { for }|j| \geqq n+1, \quad t \in \mathbb{R} .
$$

Theorem 5.4. For each nonegative integer $n$ the linear manifolds

$$
M_{n}=\left\{\zeta \in H^{s}\left(S^{1}\right) \mid \zeta_{j}=0 \text { for }|j| \geqq n+1\right\}
$$

are invariant for Eq. (5.15). On them (5.15) reduces to the ordinary differential system of $2 n+1$ complex equations obtained from (5.20) by setting $\zeta_{j}=0$ for $|j| \geqq n+1$.

Proof. We consider the expression $T(t)=\sum_{|ر| \geq n+1}\left|\zeta_{j}(t)\right|^{2}$. As long as the solution of (5.15) starting from $\zeta(0) \in H^{s}\left(S^{1}\right) \cap M_{n}$ is smooth, we derive an a priori, Gronwall type inequality

$$
T(t) \leqq T(0) \exp k(t)
$$

with $k(t)$ depending on the solution. Since $T(0)=0$ we obtain $T(t)=0$, for short times first but then we can repeat the argument, for any time, as long as the solution is finite.

In particular, for $n=1$ we obtain the system

$$
\left\{\begin{array}{l}
\dot{\zeta}_{-1}=\frac{i}{2} \zeta_{-1}-\frac{i}{2} \zeta_{-1} \zeta_{1} \\
\dot{\zeta}_{0}=\frac{i}{2} \zeta_{0} \\
\dot{\zeta}_{1}=\frac{i}{2}\left|\zeta_{-1}\right|^{2} .
\end{array}\right.
$$

Ignoring $\zeta_{0}(t)=e^{\frac{1}{2} l} \zeta_{0}$ we have the system

$$
\left\{\begin{aligned}
\dot{\zeta}_{1} & =\frac{i}{2}\left|\zeta_{-1}\right|^{2} \\
\dot{\zeta}_{-1} & =\frac{i}{2} \zeta_{-1}-\frac{i}{2} \zeta_{-1} \zeta_{1} .
\end{aligned}\right.
$$

Compare (5.21) to the exact system (3.10) governing the evolution of the Kirchhoff solutions. The system (5.21) conserves the area

$$
A=\left|1+\zeta_{1}\right|^{2}-\left|\zeta_{-1}\right|^{2}=\left|1+\zeta_{1}(0)\right|^{2}-\left|\zeta_{-1}(0)\right|^{2} .
$$

From the first equation of (5.21) and conservation of area we obtain the equation

$$
\dot{z}_{1}=\frac{i}{2}\left[\left|z_{1}\right|^{2}-\left|z_{1}(0)\right|^{2}+\left|\zeta_{-1}(0)\right|^{2}\right]
$$

for $z_{1}=1+\zeta_{1}$. From this equation we deduce that $\operatorname{Re} z_{1}=\operatorname{Re} \zeta_{1}(0)$ and that $\operatorname{Im} z_{1}=$ $\operatorname{Im} \zeta_{1}$ blows up in finite time provided $\left(\operatorname{Im}\left(\zeta_{1}(0)\right)\right)^{2}<\left|\zeta_{-1}(0)\right|^{2}$. 
Theorem 5.5. There exist initial data of arbitrarily small $H^{s}\left(S^{1}\right)$ norm for all $s \geqq 0$ such that the solution of (5.15) with those initial data becomes infinite in all $H^{s}$ spaces in finite time.

Equation (5.15) provides for short times as good an approximation of the vortex patch equation as the linear approximate equation (4.14) does. It has the advantage over the latter that it is area preserving. However, Eq. (5.15) predicts blow up in the pure modes $-1,1$ while the vortex patch equation has the Kirchhoff solutions in those modes.

\section{Nearly Circular Vortex Patches: The Quadratic Approximate Equation}

In this section we derive an equation which constitutes the second order approximation, in the vicinity of the unit circular vortex patch, of the vortex patch evolution equation. We will discuss a few exact explicit solutions of this equation.

We write the solution of the equation of evolution of vortex patches (1.13) as

$$
z(t, \alpha)=\zeta_{0}(\alpha)+\zeta(t, \alpha)
$$

with $z_{0}(\alpha)=e^{i \alpha}$. Using the fact that

$$
(i+H) z_{0}=0
$$

we obtain from (1.13) the equation for the evolution of $\zeta$ :

$$
\frac{\partial \zeta}{\partial t}(t, \alpha)=\frac{1}{2}((i+H) \zeta)(t, \alpha)+\frac{1}{2 \pi} \int_{0}^{2 \pi} \log \left|1+\frac{\zeta(t, \alpha)-\zeta(t, \beta)}{z_{0}(\alpha)-z_{0}(\beta)}\right| \frac{\partial\left(z_{0}+\zeta\right)}{\partial \beta}(t, \beta) d \beta .
$$

We expand the logarithm in the series (3.7). Discarding powers of $\zeta$ of order 3 and higher we deduce from (6.1) the quadratic approximate equation

$$
\begin{aligned}
\frac{\partial \zeta}{\partial t}(t, \alpha)= & \frac{1}{2}((i+H) \zeta)(t, \alpha)+\frac{1}{2 \pi} \int_{0}^{2 \pi} \operatorname{Re}\left(\frac{\zeta(t, \alpha)-\zeta(t, \beta)}{z_{0}(\alpha)-z_{0}(\beta)}\right) \frac{\partial\left(z_{0}+\zeta\right)}{\partial \beta}(t, \beta) d \beta . \\
& -\frac{1}{4 \pi} \int_{0}^{2 \pi} \operatorname{Re}\left(\left(\frac{\zeta(t, \alpha)-\zeta(t, \beta)}{z_{0}(\alpha)-z_{0}(\beta)}\right)^{2}\right) \frac{\partial z_{0}}{\partial \beta}(\beta) d \beta .
\end{aligned}
$$

Note that Eq. (6.2) differs from (5.15) by the quadratic term

$$
-\frac{1}{4 \pi} \int_{0}^{2 \pi} \operatorname{Re}\left(\left(\frac{\zeta(t, \alpha)-\zeta(t, \beta)}{z_{0}(\alpha)-z_{0}(\beta)}\right)^{2}\right) \frac{\partial z_{0}}{\partial \beta}(\beta) d \beta .
$$

Proposition 6.1. Assume $\zeta(0)$ belongs to $H^{s}\left(S^{1}\right)$ with $s>\frac{3}{2}$. Then there exists a unique solution $\zeta(t)$ of (6.2) having $\zeta(0)$ as initial datum for $|t| \leqq T_{0}$. The positive number $T_{0}$ depends on the norm of $\zeta(0)$ in $H^{s}\left(S^{1}\right)$.

We will not give the proof of Proposition 6.1 here. It is similar to that of Proposition 5.2, Lemma 5.3. Introducing the Fourier series representation

$$
\zeta(t, \alpha)=\sum_{j=-\infty}^{\infty} \zeta_{j}(t) e^{i j \alpha}
$$

we obtain the following infinite system of ordinary differential equations, 
equivalent to $(6.2)$ :

$$
\left\{\begin{aligned}
& \dot{\zeta}_{j}= \frac{i}{2}\left(\zeta_{j}+\bar{\zeta}_{2-j}\right)-\frac{i}{2} \bar{\zeta}_{1} \bar{\zeta}_{2-j}+\frac{i}{2} \sum_{k=-j+1}^{\infty}(k+j-1) \zeta_{k+j-1} \bar{\zeta}_{k} \\
&+\frac{i}{2} \sum_{k=-j+4}^{\infty}(k+j-3) \bar{\zeta}_{3-k-j} \bar{\zeta}_{k}-\frac{i}{2} \sum_{k=2}^{-j+1} \bar{\zeta}_{k} \bar{\zeta}_{3-k-j}, \text { for } j \leqq-1 \\
& \dot{\zeta}_{0}= \frac{i}{2}\left(\zeta_{0}+\bar{\zeta}_{2}\right)-\frac{i}{2} \bar{\zeta}_{1} \bar{\zeta}_{2}+\frac{i}{2} \sum_{k=1}^{\infty}(k-1) \zeta_{k-1} \bar{\zeta}_{k}-\frac{i}{2} \sum_{k=-\infty}^{0} k \bar{\zeta}_{k} \bar{\zeta}_{3-k}, \\
& \dot{\zeta}_{j}=-\frac{i}{2} \sum_{k=-\infty}^{-j}(j+k-1) \zeta_{j+k-1} \bar{\zeta}_{k}+\frac{i}{2} \sum_{k=-\infty}^{-j}(1-j-k) \bar{\zeta}_{3-k-j} \bar{\zeta}_{k}, \\
& \text { for } j \geqq 1 .
\end{aligned}\right.
$$

Comparing to $(5.20)$ we note that the term

$$
-\frac{1}{4 \pi} \int_{0}^{2 \pi} \operatorname{Re}\left(\left(\frac{\zeta(t, \alpha)-\zeta(t, \beta)}{z_{0}(\alpha)-z_{0}(\beta)}\right)^{2}\right) \frac{\partial z_{0}}{\partial \beta}(\beta) d \beta
$$

added several new terms to (5.20), some of which cancelled existing terms in (5.20); all but one of these new terms still possess the property that the dummy variable $k$ is restricted to the tail $|k| \geqq|j|$.

The exceptional term

$$
-\frac{i}{2} \sum_{k=2}^{-j+1} \bar{\zeta}_{k} \bar{\zeta}_{3-k-j}
$$

appears in the equations for the coefficients $\zeta_{j}$ with $j \leqq-1$. Although it is a lower order term in some sense (no large coefficients depending on $k$ are involved) its presence precludes the invariance of the manifolds $M_{n}$ (see Proposition 5.4) for $n \geqq 4$.

The remainder of this section is devoted to a few exact solutions of (6.2).

First we study the solutions of the form

$$
\zeta(t, \alpha)=\zeta_{1}(t) e^{i \alpha}+\zeta_{-1}(t) e^{-i \alpha}
$$

Substituting in (6.2) or (6.3) we see that the structure (6.4) is preserved by the equation provided that the coefficients $\zeta_{1}(t), \zeta_{-1}(t)$ satisfy the system

$$
\left\{\begin{aligned}
\dot{\zeta}_{1} & =\frac{i}{2}\left|\zeta_{-1}\right|^{2} \\
\dot{\zeta}_{-1} & =\frac{i}{2} \zeta_{-1} .
\end{aligned}\right.
$$

This system is an approximation, up to cubic terms, of the system (3.10) governing the evolution of the exact Kirchhoff solutions. Actually the second equation in (6.5) is identical with that in (3.10). The solutions to (6.5) do not blow up in contrast to the solutions to (5.21), the area preserving approximation. The solutions to (6.5) are

$$
\zeta(t, \alpha)=\left(\zeta_{1}(0)+\frac{i}{2}\left|\zeta_{1}(0)\right|^{2} t\right) e^{i \alpha}+\zeta_{-1}(0) e^{(i / 2) t} e^{-i \alpha}
$$


Comparing $e^{i \alpha}+\zeta(t, \alpha)$ to the exact Kirchhoff solution with the same initial data given in (3.11)

$$
z(t, \alpha)=\left(1+\zeta_{1}(0)\right) e^{(i / 2)\left(\left.\zeta_{-1}(0)\right|^{2} / 1+\left.\zeta_{1}(0)\right|^{2}\right) t} e^{i \alpha}+\zeta_{-1}(0) e^{(i / 2) t} e^{-i \alpha},
$$

we obtain that

$$
\left|e^{i \alpha}+\zeta(t, \alpha)-z(t, \alpha)\right|=O\left(\varepsilon^{2} t\right),
$$

where $\varepsilon$ is the size of the disturbances $\zeta_{1}(0), \zeta_{-1}(0)$. Thus the solutions to the quadratic approximate equation (6.2) yield approximate vortex patches which are good long time approximations to the known exact solutions (3.11). They are in better agreement with the Kirchhoff solutions over long times than the solutions of the area preserving equations (5.15), (5.21).

Somewhat surprising is the fact that the ansatz

$$
\zeta(t, \alpha)=\zeta_{1}(t) e^{i \alpha}+\zeta_{-m}(t) e^{-i m \alpha}
$$

for $m \geqq 2$ is also consistent with the quadratic approximate equation (6.2). The system we obtain for the coefficients $\zeta_{1}, \zeta_{-m}$ is

$$
\left\{\begin{array}{c}
\dot{\zeta}_{1}=\frac{i m}{2}\left|\zeta_{-m}\right|^{2} \\
\dot{\zeta}_{-m}=\frac{i}{2} \zeta_{-m} .
\end{array}\right.
$$

Therefore we proved

Proposition 6.2. For every $m \geqq 1$ the functions

$$
\zeta^{(m)}(t, \alpha)=\left(\zeta_{1}(0)+\frac{i m t}{2}\left|\zeta_{-m}(0)\right|^{2}\right) e^{i \alpha}+\zeta_{-m}(0) e^{i t / 2} e^{-i m \alpha}
$$

are solutions of (6.2).

The family $\zeta^{(m)}$ shows that Eq. (6.3) cannot be well posed in $H^{s}\left(S^{1}\right)$ for $s<\frac{1}{2}$. However, for $s \geqq \frac{1}{2}$ one has

$$
\left\|\zeta^{(m)}(t, \cdot)\right\|_{s} \leqq \sqrt{2}\left\|\zeta^{(m)}(0)\right\|_{s}+\frac{t}{\sqrt{2}}\left\|\zeta^{(m)}(0)\right\|_{1 / 2}^{2} .
$$

The space $H^{1 / 2}\left(S^{1}\right)$ plays naturally a special role: it is both a critical Sobolev imbedding space and the space which provides natural sufficient conditions for the area functional $A(\zeta)$ (see (5.4)) to be defined.

As we mentioned earlier the linear manifolds $M_{n}$ for $n \geqq 4$ are not invariant for Eq. (6.2) (in contrast to Eq. (5.15)). However $M_{3}, M_{2}$ and $M_{1}$ are invariant.

Proposition 6.3. The 7 complex dimensional linear manifold

$$
M_{3}=\left\{\zeta \in H^{1}\left(S^{1}\right) \mid \zeta=\sum_{j=-3}^{3} \zeta_{j} e^{i j \alpha}, \zeta_{j} \in \mathbb{C}\right\}
$$


is invariant for (6.2). On it the equation reduces to the system of 7 complex ordinary differential equations obtained from (6.3) by setting $\zeta_{j}=0$ for $|j| \geqq 4$,

$$
\left\{\begin{array}{l}
\dot{\zeta}_{-3}=\frac{i}{2} \zeta_{-3}-\frac{i}{2}\left(\bar{\zeta}_{3}\right)^{2} \\
\dot{\zeta}_{-2}=\frac{i}{2} \zeta_{-2}-i \bar{\zeta}_{2} \bar{\zeta}_{3} \\
\dot{\zeta}_{-1}=\frac{i}{2} \zeta_{-1}+\frac{i}{2} \bar{\zeta}_{3}+\frac{i}{2} \zeta_{1} \bar{\zeta}_{3}-\frac{i}{2} \bar{\zeta}_{1} \bar{\zeta}_{3}-\frac{i}{2}\left(\bar{\zeta}_{2}\right)^{2} \\
\dot{\zeta}_{0}=\frac{i}{2} \zeta_{0}+\frac{i}{2} \bar{\zeta}_{2}+\frac{i}{2} \zeta_{1} \bar{\zeta}_{2}+i \zeta_{2} \bar{\zeta}_{3}-\frac{i}{2} \bar{\zeta}_{1} \bar{\zeta}_{2} \\
\dot{\zeta}_{1}=\frac{i}{2}\left[\left|\zeta_{-1}\right|^{2}+2\left|\zeta_{-2}\right|^{2}+3\left|\zeta_{-3}\right|^{2}\right]+\frac{i}{2} \bar{\zeta}_{-1} \bar{\zeta}_{3} \\
\dot{\zeta}_{2}=\frac{i}{2} \zeta_{-1} \bar{\zeta}_{-2}+i \zeta_{-2} \bar{\zeta}_{-3}+\frac{i}{2} \bar{\zeta}_{-2} \bar{\zeta}_{3} \\
\dot{\zeta}_{3}=\frac{i}{2} \zeta_{-1} \bar{\zeta}_{-3}+\frac{i}{2} \bar{\zeta}_{-3} \bar{\zeta}_{3} .
\end{array}\right.
$$

Note that setting $\zeta_{0}=\zeta_{2}=\zeta_{-2}=\zeta_{3}=\zeta_{-3}=0$ we get the system (6.5) as an invariant subsystem of (6.11), setting $\zeta_{2}=\zeta_{3}=\zeta_{-3}=\zeta_{0}=\zeta_{-1}=0$ we get (6.9) for $m=2$ and setting $\zeta_{0}=\zeta_{-1}=\zeta_{2}=\zeta_{-2}=\zeta_{3}=0$ we get (6.9) for $m=3$. The invariance of $M_{2}$ follows by observing that setting $\zeta_{3}=\zeta_{-3}=0$, we obtain an invariant subsystem of (6.11).

Acknowledgements. It is our pleasure to thank Russel Caflisch and Andrew Majda for interesting and stimulating discussions. P. Constantin acknowledges a Sloan research fellowship and the partial support of the National Science Foundation under NSF grant DMS 860-2031. E. S. Titi was partially supported by DOE grant DE-FG02-86ER25020 and by NSF grant DMS $850-7784$.

\section{References}

1. Constantin, P., Lax, P. D., Majda, A.: A simple one-dimensional model for the three-dimensional vorticity equation. Commun. Pure Appl. Math. 38, 715-724 (1985)

2. Constantin, P., Majda, A.: The Beltrami spectrum for incompressible fluid flows. Commun. Math. Phys. 115, 435-456 (1988)

3. Dritschel, D. G.: The stability and energetics of corotating uniform vortices. J. Fluid Mech. 157, 95134 (1985)

4. Lamb, H.: it Hydrodynamics, Dover, 1945

5. Majda, A.: Vorticity and the mathematical theory of incompressible fluid flow. Commun. Pure Appl. Math. 39, 187-S220 (1986)

6. Mushkelishvili, N. T.: Singular integral equations. Groningen: P. Noordhoff N.V. 1985

7. Wan, Y. H., Pulvirenti, M.: Nonlinear stability of circular vortex patches. Commun. Math. Phys. 99, 435-450 (1985) 
8. Yudovitch, V. I.: Non-stationary flow of an ideal incompressible liquid. Zh. Vych. Mat. 3, 1032-1066 (1963) (In Russian)

9. Zabusky, N., Hughes, M. H., Roberts, K. V.: Contour dynamics for the Euler equations in two dimensions. J. Comp. Phys. 30, 96-106 (1979)

Communicated by C. H. Taubes

Received August 21, 1987; in revised form May 24, 1988 